\title{
CENTROS DE APOIO FAMILIAR E ACONSELHAMENTO PARENTAL: PROPOSTA DE UM MODELO GLOBAL DE ORGANIZAÇÃO ${ }^{1}$
}

\author{
Ana Teixeira de Melo e Madalena Alarcão \\ Universidade de Coimbra, Coimbra, Portugal
}

\begin{abstract}
RESUMO: Os Centros de Apoio Familiar e Aconselhamento Parental constituem, no panorama dos serviços sociais portugueses, uma tipologia relativamente recente de serviços de apoio familiar dirigidos a crianças e jovens em situação de risco e suas famílias. No entanto, tendem a operar de formas muito distintas, carecendo ainda de enquadramento técnico e legal adequado. No presente artigo, revêem-se os objetivos dos CAFAP e apresenta-se uma proposta de um Modelo Global de Organização destes serviços, para que se apresentem como recursos eficazes de apoio ao trabalho solicitado pelos Tribunais e pelas Comissões de Protecção de Crianças e Jovens, bem como de suporte à própria comunidade, na protecção e promoção do bem-estar das famílias, crianças e jovens. O modelo apresentado pretende oferecer um enquadramento de base que permita uniformizar práticas e metodologias e facilitar o desenvolvimento de esforços futuros de investigação e avaliação da eficácia dos serviços prestados.
\end{abstract}

PALAVRAS-CHAVE: crianças em risco; serviços centrados na família; intervenção familiar; comunidade; avaliação psicológica; maus tratos.

\section{THE CENTERS FOR FAMILY SUPPORT AND PARENTAL COUNSELING: PROPOSAL OF A GLOBAL ORGANIZATION MODEL}

\begin{abstract}
The Centros de Apoio Familiar e Aconselhamento Parental (CAFAP, Center for Family Support and Parental Counseling) correspond, in the Portuguese reality of Portuguese services, to a category of services designed to support families with at-risk children and youth. However, these services tend to operate in many different ways, still without legal and technical framing. In the present article, one aims to review the CAFAP's objectives and present a Global Organization Model for these services, so they can operate as effective support resources for the activities of the courts, the child protection services and the community in the protection and promotion of families', children's and youth's well-being. With the purpose of creating common practices and methodologies, the proposed model offers a basic framework for the development of future research and evaluation efforts in regard to the efficacy of the services provided.
\end{abstract}

KEYWORDS: at-risk children; family-focused services; family intervention; community; psychological assessment; maltreatment.

\section{Introdução}

Os serviços de apoio a famílias têm já um percurso e uma história consideráveis (Manalo \& Meezan, 2000). De acordo com a definição de Hoagwood (2005), os serviços focados na família incorporam uma grande variedade de intervenções disponibilizadas às famílias, ou por elas dinamizadas, relativamente aos cuidados proporcionados às crianças. Se é verdade que começam a surgir centros familiares que oferecem um leque muito alargado de serviços e que procuram combater a estigmatização, evitando encaminhamentos para especialistas e oferecendo intervenções para famílias com características muito diversas (WarrenAdamson, 2006), por outro lado, diferentes populações apresentam diferentes necessidades, pelo que pode ser importante distinguir e disponibilizar às comunidades distintas modalidades de intervenção, com diferentes formatos e intensidades, organizando-as num contínuo, em função do risco a que as crianças e as famílias estão expostas (McCroskey \& Meezan, 1998; Scannapieco $\&$ Connel-Corrick, 2005).

$\mathrm{O}$ conceito de risco tem-se revestido na literatura de alguma ambiguidade, sofrendo diversas alterações e assumindo significados e conotações diferentes ao longo do tempo (Hillesheim \& Cruz, 2008). Para efeitos de clarificação, consideramos, neste artigo, o risco como a probabilidade de ocorrência de desajustamento futuro (Werner \& Smith, 1992), assumindo que o mesmo é melhor percebido num contínuo (Rutter, 2005). Neste 
sentido, as crianças e jovens em risco são aqueles em cujas trajetórias desenvolvimentais é possível identificar um conjunto de factores de natureza constitucional e/ou ambiental que aumentam a probabilidade de desajustamento ou aparecimento de perturbações futuras (Werner \& Smith, 1992). Pelo contrário, crianças e jovens em perigo são aqueles que estão expostos a situações que podem afetar, no imediato, de forma séria e grave, a sua integridade física e/ou psicológica. O risco implica, assim, uma visão alargada no tempo e a eventualidade (probabilidade) de aparecimento de problemas futuros, enquanto o perigo se refere ao aparecimento de danos imediatos, incluindo-se algumas das situações previstas, em Portugal, pela Lei de Protecção de Crianças e Jovens em Perigo (Portugal, ano?), nomeadamente o fato de a criança estar abandonada ou entregue a si própria, sofrer de maus tratos físicos ou psíquicos ou ser vítima de abusos sexuais, não ter os cuidados ou a afeição adequados à sua idade e situação pessoal, ser obrigada a trabalhos excessivos, estar sujeita a comportamentos que afetam gravemente e sua segurança ou equilíbrio emocional, assumir comportamentos ou envolver-se em atividades que afectem gravemente a sua segurança, formação, educação ou desenvolvimento.

As perspectivas ecológicas (Bronfenbrenner, 1979) contribuíram para que os serviços centrados na família diversificassem o tipo de intervenções considerando diversos níveis de influências contextuais que interagem entre si, e com as características da criança, na construção dos seus percursos desenvolvimentais.

Vários tipos de intervenções centradas em famílias com crianças e jovens em risco, ou perigo, nomeadamente de maltrato, têm vindo a ser estudadas e implementadas, como é o caso de diferentes correntes de terapia familiar, programas de intervenção intensiva para preservação da família, gestão de casos, programas compreensivos e multimodais, programas de treino de competências parentais ou intervenções de suporte social (Berry, Charlson, \& Dawson, 2003; Chaffin \& Friedrich, 2004; Dufour \& Chamberland, 2004). Os programas distinguem-se uns dos outros em termos das variáveis alvo e metodologias utilizadas. Alguns programas mais intensivos, no domicílio, tendem a estar associados aos serviços de preservação da família (tendo em vista evitar a retirada de uma criança), enquanto as modalidades de terapia familiar apresentam um enfoque maior nas dinâmicas relacionais (Carr, 2000). Os programas de treino de competências parentais centram-se em diferentes dimensões da capacidade parental associadas a uma melhor prestação de cuidados às crianças, seja nas dimensões relacionadas com os cuidados básicos, com a supervisão ou com a dimensão afetiva, seja na orientação e estabelecimento de limites (Barlow,
Johnston, Kendrick, Polnay, \& Stewart-Brown, 2006; Taylor \& Biglan, 1998), enquanto o enfoque de outros programas é feito na qualidade da interacção pais-filhos (Eyberg, 2003). Algumas intervenções apresentam um carácter multimodal e incorporam diferentes estratégias (Lutzker \& Bigelow, 2002), podendo abranger pais e filhos (Donohue \& Van Hasselt, 1999), vários contextos e outros sistemas para além da família (Barth et al., 2007). Os programas diferem na medida em que se baseiam em protocolos de intervenção estruturados, mais comuns nas abordagens de cariz comportamental, ou em linhas orientadoras que permitem desenhar planos de intervenção específicos para cada família, mais frequentes nas abordagens compreensivas, terapia familiar e programas de intervenção no domicílio.

As intervenções terapêuticas multifamiliares, originárias do campo do tratamento da doença mental (Asen, 2002), têm sido adaptadas a outros contextos e proliferado sob a forma de intervenções psicoeducativas familiares em grupo (Hughes, 1994; Murray-Swank \& Dixon, 2004). Os programas de educação parental e treino de competências parentais ou familiares (Bunting, 2004) partilham muitos dos princípios da terapia multifamiliar, com maior ênfase ora no ensino e treino de competências ora na partilha de experiências e no suporte emocional e social. Regra geral estas intervenções têm efeitos positivos adicionais ao nível da redução do isolamento das famílias e stress parental.

Os programas de suporte social e de gestão de casos tendem a focar-se na aquisição e gestão de recursos. Autores como Rojano $(2000,2004)$ sublinham a necessidade de, com famílias pobres, a intervenção ter em atenção o desenvolvimento individual e familiar e ser acompanhada de intervenção socio-económica. Nos últimos anos, a intervenção familiar tem vindo a extrapolar os limites da família, reforçando-se não só o seu envolvimento cívico, as suas capacidades de liderança e aliança com sistemas mais fortes que a podem apoiar no seu desenvolvimento (Rojano) mas também a emergência de intervenções resultantes de parcerias comunitárias em que as famílias são implicadas desde a avaliação de necessidades à planificação e implementação de atividades que permitam promover o bem-estar dos elementos da família, numa dada comunidade (Doherty \& Beaton, 2000). Verifica-se, deste modo, uma tendência não só para a integração de intervenções sociais e clínicas mas, também, comunitárias (Costa \& Brandão, 2005).

Biglan e Smolkowski (2002) lançaram, para o séc. XXI, vários desafios: apoiar as comunidades no desenvolvimento de objectivos e estratégias para o seu fortalecimento, monitorizando o seu bem-estar; o de estabelecer uma ponte entre a prática e a investigação, 
os profissionais do terreno e o mundo acadêmico; o de apostar na formação dos profissionais para que estes possam implementar intervenções mais eficazes. É nossa posição que também os serviços de apoio às famílias, em articulação com outras instituições comunitárias, deveriam ter estes objetivos presentes.

As últimas décadas foram marcadas pela emergência da prevenção, tendo sido advogada a necessidade não só de prevenir o aparecimento de problemas futuros, diminuindo os factores de risco a que as crianças estão expostas e aumentando os factores protetores, como de promover o seu bem-estar de uma forma mais alargada (Mrazek \& Haggerty, 1994; Weissberg, Kumpfer, \& Seligman, 2003). A este nível, tem-se assistido ao desenvolvimento e avaliação da eficácia de vários programas de prevenção focados na família e que visam o seu fortalecimento, existindo já um conjunto diversificado de propostas com evidência científica em diversas áreas (Kumpfer \& Alvarado, 2003), nomeadamente na prevenção dos maus tratos infantis e negligência (Lundahl, Nimer, \& Parsons, 2006). As posições mais atuais propõem que os programas de prevenção possam ser categorizados em três níveis, em função das características da população abrangida (Mrazek \& Haggerty, 1994). Fala-se, assim, de prevenção universal quando os programas se destinam à população em geral, de prevenção seletiva quando dirigida a grupos em que são identificados determinados factores de risco e de prevenção indicada para população de alto-risco ou população em que se verificam já indícios ou precursores próximos dos problemas que se pretende prevenir. Os serviços de apoio à família, novamente em articulação com outros, devem assumir a prevenção, nos diferentes níveis, como uma preocupação central. Em Portugal, a prevenção é, aliás, uma das áreas de trabalho sugeridas para as Comissões Alargadas das Comissões Nacionais de Protecção de Crianças e Jovens (CPCJ), com as quais, todos os serviços de apoio à família, inclusivamente os CAFAP, devem colaborar.

Os Centros de Apoio Familiar e Aconselhamento Parental (CAFAP), relativamente recentes, em Portugal, e definidos como uma

resposta social, desenvolvida através de um serviço, vocacionada para o estudo e prevenção de situações de risco social e para o apoio a crianças e jovens em situação de perigo e suas famílias, concretizado na sua comunidade, através de equipas multidisciplinares (Direcção Geral da Segurança Social, da Família e da Criança [DGSSFC], 2006),

constituem, assim, um serviço de apoio a famílias com crianças e jovens em situação de perigo ou risco social. Estes serviços apresentam, de acordo com a Segurança Social (DGSSFC, 2006) os seguintes objetivos: promover o estudo e a avaliação de famílias em risco psicossocial; prevenir situações de perigo; evitar rupturas que possam levar à institucionalização; assegurar a satisfação das necessidades físicas, cognitivas, emocionais e sociais das crianças e jovens; reforçar as competências pessoais dos intervenientes no sistema familiar das crianças e jovens através de uma abordagem integrada dos recursos da comunidade; promover a mediação entre a família e os serviços envolvidos para facilitar a comunicação, potenciar contactos e promover a solução de eventuais dificuldades; contribuir para a autonomia das famílias.

Podendo constituir-se, como entidades com competência em matéria de infância e juventude (Lei de Protecção de Crianças e Jovens em Perigo), os CAFAP poderiam apresentar-se como um recurso importante de apoio às $\mathrm{CPCJ}$, aos Tribunais e aos serviços sociais mais tradicionais, prestando serviços integrados de caráter simultaneamente terapêutico, educativo e social, se fosse desenhada uma linha orientadora comum dos seus procedimentos de avaliação e intervenção e avaliada a sua eficácia. Poderiam constituir-se como entidades de suporte quer ao nível da avaliação quer em termos da operacionalização das medidas de apoio junto dos pais.

No entanto, os CAFAP operam com formas de funcionamento muito distintas, sem uma estrutura ou linha orientadora comum que permita não só a uniformização de algumas práticas como uma avaliação mais consistente da sua eficácia, não dispondo, ainda, de enquadramento técnico e legal que delimite o seu funcionamento. Se, por um lado, os serviços podem organizar-se, de forma mais ou menos autônoma e criativa, procurando ir ao encontro das necessidades da população que servem, por outro lado, corre-se o risco de subaproveitar um recurso importante que poderia assumir funções complementares relativamente a outros serviços e respostas sociais já existentes, pela eventual falta de sistematização, consistência, continuidade das intervenções e adequada avaliação.

É nesse sentido que apresentamos uma proposta de organização para os CAFAP, em termos da definição de objetivos, população-alvo, níveis e modalidades de intervenção esperando poder contribuir para a melhoria dos serviços prestados, para a optimização dos recursos existentes e para uma melhor avaliação futura dos serviços realizados.

\section{Proposta de modelo global de organização para os CAFAP}

\section{Princípios orientadores}

Embora a definição dos CAFAP, apresentada pela Segurança Social (DGSSFC, 2006) e anteriormente 
referida, contemple a possibilidade de separação entre a intervenção com as crianças/ jovens, por um lado, e com as famílias, por outro, quando refere que o serviço se destina ao "apoio de crianças e jovens em situação de perigo e suas famílias", consideramos prioritário assumir que os mesmos têm como finalidade o apoio a famílias com crianças e jovens em situação de risco ou de perigo. Desta forma, colocamos o centro da atenção na família, ainda que a pretexto da criança. Muito embora o trabalho com famílias seja frequente, em Portugal, não só nos CAFAP como noutros serviços de apoio familiar, nem sempre se concretiza num trabalho focado e guiado por uma visão centrada na família e por uma grelha de leitura sistêmica. Como consequência, as famílias podem continuar a receber serviços desgarrados, centrados nos indivíduos que a compõem, mais do que nas suas dinâmicas relacionais e nos padrões de interação, e focalizados nas narrativas familiares e contextos que contribuem para, ou mantêm, situações problemáticas. Uma leitura sistêmica pode minimizar o risco de se procurar produzir mudanças nos indivíduos (por exemplo, nos comportamentos da criança ou nos comportamentos dos prestadores de cuidados) sem se ter em consideração a possibilidade de estes comportamentos interagirem com outros e com outras dimensões do funcionamento familiar. Deste modo, podem evitar-se os perigos da manutenção de olhares, centrados no indivíduo, que sustentam situações de exclusão, estigmatização ou culpabilização da família, atrofiadoras do seu desenvolvimento positivo. Por outro lado, quando existem equipas multidisciplinares, uma perspectiva sistémica pode diminuir o risco de se desenvolverem intervenções segmentadas, realizadas por diferentes profissionais, sem a devida integração e consideração pelo caráter holístico e pela coerência do funcionamento humano e da família, tendo como resultado um somatório desintegrado de diferentes intervenções (Matos \& Sousa, 2004). Advoga-se, assim, à semelhança do que tem sido proposto por outros autores, um modelo centrado e guiado pela família, com um caráter verdadeiramente multidisciplinar, em que diferentes olhares não só se complementam como se complexificam e flexibilizam mutuamente, resultando na definição de ações partilhadas e complementares que se unem, em vez de caminharem em linhas paralelas que nunca se cruzam verdadeiramente.

Existem vários serviços que, não sendo específi$\cos$ (e.g., centros comunitários, serviços de atendimento/acompanhamento social; equipas de Rendimento
Social de Inserção), prestam, frequentemente, apoio a famílias com crianças e jovens maltratadas, em situação de risco ou perigo. Os CAFAP devem assumir uma identidade diferenciada de outras tipologias de serviços de apoio à família e comunidade ou à criança e jovem em perigo, oferecendo intervenções distintas das que são habitualmente proporcionadas por aqueles. Por outro lado, dados os custos associados a este tipo de respostas, considera-se pertinente apostar em serviços que vão para além do acompanhamento individual passível de ser prestado por serviços públicos e privados (como a psicoterapia ou o aconselhamento individual), do apoio social básico ou de formas de terapia familiar tradicionais. Os CAFAP devem poder assegurar serviços de qualidade, devidamente fundamentados em termos teóricos e intencionalizados, dirigidos privilegiadamente a situações que, pela sua complexidade, não encontram facilmente respostas noutras instituições comunitárias e que exigem uma atenção especializada.

Propõe-se, então, que os CAFAP: (a) se assumam como serviços orientados para a família mantendo presentes as necessidades de segurança e bem-estar da criança e do jovem; (b) se orientem por modelos ecológico-desenvolvimentais e sistêmicos e valorizem modelos de resiliência familiar e individual, de forma a centrarem-se nas forças e no reforço de competências relacionais e individuais necessárias para a construção do bem-estar das crianças e jovens, no presente e no futuro; (c) privilegiem a construção de relações de colaboração com as famílias, promotoras da autonomia e empowerment; (d) promovam a articulação e colaboração com a comunidade e com as instituições comunitárias.

\section{Objetivos}

Propõe-se uma definição de objetivos complementares aos apresentados pela Direcção Geral de Segurança Social, da Família e da Criança, organizados em diferentes níveis de integração e grau de especificidade a partir dos quais é possível (a) definir uma orientação global e um sentido de missão, no nível mais integrador, (b) resultados globais esperados, nos níveis intermédios, e (c) campos específicos de atuação nos níveis inferiores, que permitem a concretização dos níveis superiores e a definição de indicadores de avaliação claros e mensuráveis. A estes objetivos correspondem diferentes eixos de intervenção e populações, conforme apresentado na Figura 1. 
Figura 1- Relação entre objetivos, eixos de intervenção e população-alvo

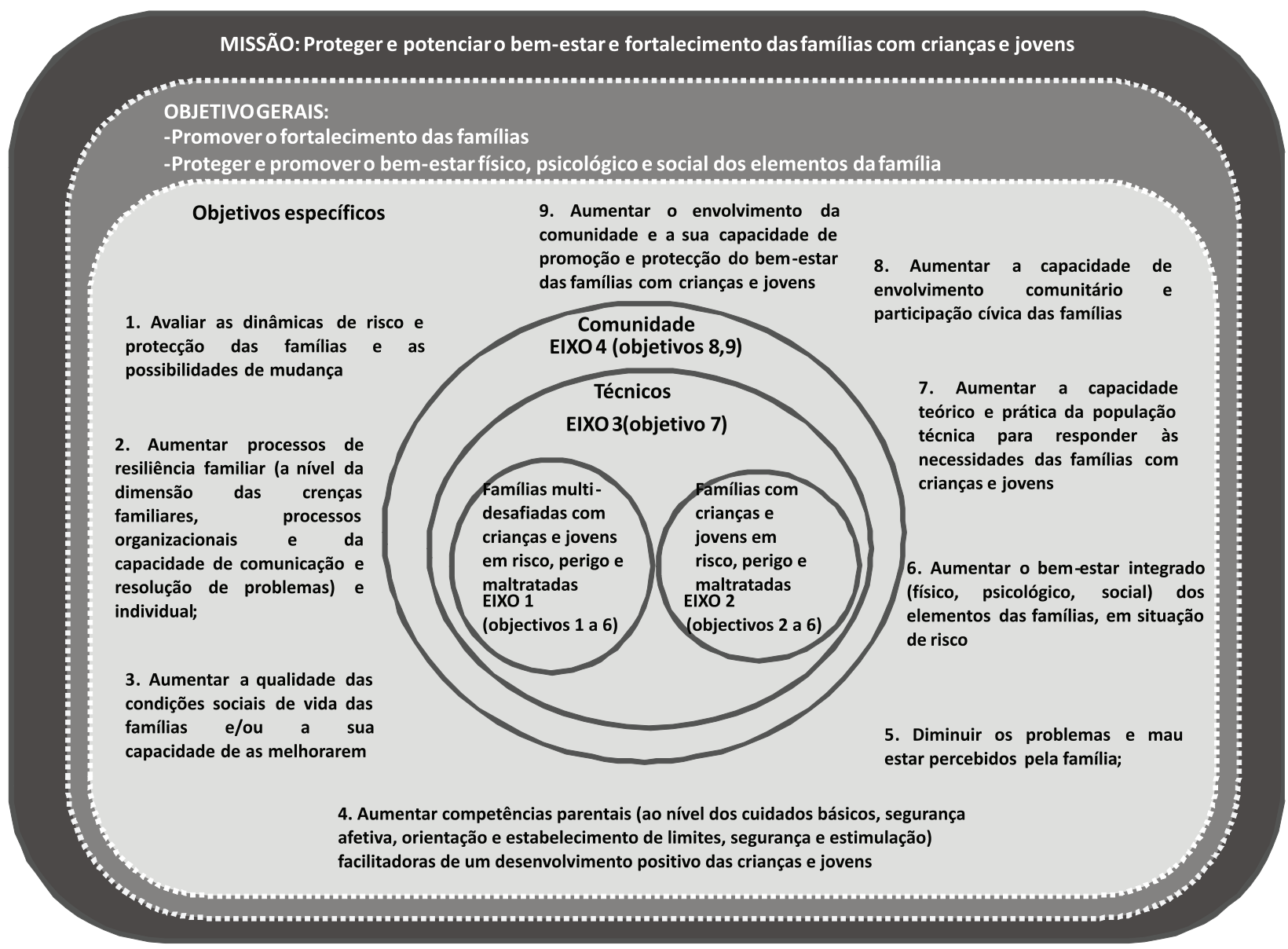

É, assim, proposto que o sentido de missão se defina da seguinte forma: proteger e potenciar o bemestar e fortalecimento das famílias com crianças e jovens em risco ou perigo. Como objetivos gerais propõem-se, para os CAFAP, os seguintes: 1) promover o fortalecimento das famílias; 2) proteger e potenciar o bem-estar físico, psicológico e social dos elementos das famílias. Em termos de objetivos especificos assumem-se os seguintes: (a) avaliar as dinâmicas de risco e protecção das famílias e as possibilidades de mudança; (b) aumentar processos de resiliência familiar (a nível da dimensão das crenças familiares, processos organizacionais e capacidade de comunicação e resolução de problemas) e individual; (c) aumentar a qualidade das condições sociais de vida das famílias e/ou a sua capacidade de as melhorarem; (d) aumentar competências parentais (ao nível dos cuidados básicos, segurança afetiva, orientação e estabelecimento de limites, segurança e estimulação) facilitadoras de um desenvolvimento positivo das crianças e jovens; (e) diminuir os problemas e mau estar percebidos pelas famílias; (f) aumentar o bem-estar integrado (físico, psicológico, social) dos elementos das famílias, em situação de risco; (g) aumentar a capacida- de teórica e prática da população técnica para responder às necessidades das famílias com crianças e jovens em situação de risco ou perigo; (h) aumentar a capacidade de envolvimento comunitário e participação cívica das famílias; (i) aumentar o envolvimento da comunidade e a sua capacidade de promoção e proteção do bem-estar das famílias com crianças e jovens.

\section{População-Alvo}

Propõe-se que a população-alvo dos CAFAP compreenda, privilegiadamente, famílias com crianças e jovens em situação de risco ou perigo, nomeadamente de maltrato. Complementarmente, a comunidade em geral, incluindo a comunidade técnica, pode constituir-se como população-alvo das ações dos CAFAP, ainda que num plano essencialmente estratégico, ou intermédio.

\section{Organização em eixos de intervenção}

Para alcançar os objetivos acima referidos, apresenta-se uma proposta de organização global dos CAFAP estruturada em níveis diferenciados de intervenção que se agrupam de acordo com as modalidades de intervenção e/ou a população-alvo. 


\section{Eixo 1: Avaliação e intervenção familiar integrada}

O eixo 1 pretende dar resposta aos objetivos especificos (a) a (f) anteriormente apresentados, assumindo como população-alvo, direta, famílias multidesafiadas com crianças e jovens maltratados ou em situação de risco e traduz-se na implementação do Modelo de Avaliação e Intervenção Familiar Integrada (MAIFI) (Melo \& Alarcão, 2008).

O eixo 1 é particularmente indicado para famílias em que ocorreu uma situação de mau trato ou negligência e/ou famílias afetadas por múltiplos problemas ou famílias multi-desafiadas. Na verdade, a família que maltrata enfrenta, frequentemente, múltiplos problemas (Cirillo \& DiBlasio, 1997). Pelas caraterísticas dos serviços prestados, este eixo pode, ainda, dirigir-se especialmente a situações em que a retirada da criança do seio familiar é eminente, mas se julga possível, ainda, intervir, preservando-a na família, e situações em que a criança está acolhida e é necessário avaliar e/ou apoiar a família para que o processo de reunificação possa acontecer ou para que dele se possa desistir em definitivo.

Não se considerando existir situações verdadeiramente contra-indicadas para este eixo, pensa-se que algumas podem ser pouco indicadas, em particular situações de famílias com problemas relativamente focalizados cujas necessidades podem ser facilmente satisfeitas por outras respostas, não existindo necessidade de uma avaliação e intervenção exaustiva e em diferentes áreas, nem de um acompanhamento muito intensivo e próximo. A intervenção, neste eixo, traduzida na implementação do MAIFI, caracteriza-se por ser muito focalizada e intensiva visando, por um lado, a avaliação do potencial de mudança das famílias e das condições socio-familiares e de vida dos menores bem como a produção de informação e emissão de pareceres que facilitem o processo de tomada de decisão quanto às ações a adotar para salvaguarda da segurança das crianças e jovens. Por outro lado, visa o desenho e implementação de planos de intervenção definidos à medida de cada família, que podem ter como finalidade prevenir a retirada dos menores e/ou promover a reunificação familiar. A intervenção, bastante intensiva e sistemática, deve ser conduzida por uma equipa constituída por um mínimo de dois profissionais, sendo um necessariamente psicólogo e outro um profissional da área social (assistente social, educador social, etc.). O trabalho decorre com privilégio do espaço domiciliário e comunitário em que a família se movimenta e com/ sobre os contextos circundantes e em horários convenientes para a família, usando diferentes estratégias para facilitar o envolvimento e manutenção da mesma ao longo do processo.

\section{Eixo 2: Intervenção psicoeducativa multifamiliar}

$\mathrm{O}$ eixo 2 foca-se nos objetivos específicos (b) a (f), conforme anteriormente descrito, assumindo como população-alvo, específica, famílias com crianças e jovens maltratados ou em situação de risco.

São particularmente indicadas para este eixo famílias em que existem problemas ou vulnerabilidades relativamente focalizados em áreas específicas do funcionamento familiar, estando contra-indicadas situações de famílias multi-desafiadas que enfrentam dificuldades em várias áreas do funcionamento familiar. Podem ser indicadas para este eixo situações em que, não existindo dificuldades evidentes instaladas ao nível do funcionamento familiar, se verificam um conjunto de condições psicossociais (e.g., desemprego de longa duração; história de abuso de substâncias na família; pobreza; residência em zonas de elevada criminalidade ou violência, etc.) e dúvidas ou dificuldades na gestão da parentalidade que podem colocar a família em risco. Pode, então, ser particularmente benéfico aumentar factores de proteção, nomeadamente ao nível da relação pais-filhos. Neste eixo podem, ainda, ser incluídas famílias de médio e baixo risco para a ocorrência de maus tratos. Sabe-se que o risco aumenta em situações em que há um percurso de maus tratos prolongados, história de maus tratos dos próprios pais, violência conjugal, doença mental, envolvimento em atividades ilícitas e consumo de substâncias, idade precoce da criança, problemas de comportamento ou deficiência da criança e quando o prestador de cuidados culpa a criança por algum incidente de abuso físico, emocional ou negligência (Righthand, Kerr, \& Drach, 2003). Estes factores devem assim ser tidos em consideração quando se pondera a integração de uma família numa intervenção multifamiliar. Dependendo das características dos padrões interacionais, das crenças e processos narrativos dos sistemas familiares a intervenção estandardizada em grupo poderá não ser suficiente ou mesmo adequada. Por conseguinte, e, por exemplo, no caso de se tratar de famílias expostas a múltiplas fontes de perturbação e problemas, em que o mau trato emerge, sozinho ou acompanhado por outros problemas, como sintoma de dificuldades de relacionamento conjugais, com a família de origem, ou de problemas sociais, esta pode não ser a modalidade mais aconselhada. É importante ter em consideração que, por vezes, a incapacidade parental, o mau trato ou inadaptação familiar pode ser entendida como sintoma de um sistema que luta para encontrar formas alternativas de funcionamento (Cirillo \& DiBlasio, 1997) e, nesse caso, é importante pensar se o programa em questão poderá permitir desencadear processos de mudança eficazes.

No eixo 2 poderão ser desenvolvidas atividades de intervenção em grupo ou projetos de prevenção 
centrados na família, como programas de educação parental, projectos de treino de competências parentais ou familiares, programas de auto-ajuda, programas centrados no suporte social, programas de treino de interação pais-filhos. Para além de assentar num formato de grupo, a intervenção deste eixo é caracterizada por ser bastante focalizada e não implicar uma avaliação exaustiva prévia à intervenção. Não obstante possa existir alguma flexibilidade, a intervenção tende a ter uma duração pré-definida e a ser relativamente estandardizada. Por outro lado, apresenta um cariz mais educativo do que terapêutico.

Deve ser dada prioridade a programas já avaliados e baseados em evidências científicas, preferencialmente os que englobem toda a família, ter em consideração os mecanismos de risco e protecção e incidir sobre processos chave de resiliência familiar. Importa ter em consideração que a intervenção nestes casos é mais limitativa do que a anterior e que não parte, necessariamente, de uma avaliação aprofundada das dinâmicas familiares.

\section{Eixo 3: Serviços voltados para a comunidade}

$\mathrm{O}$ eixo 3 está focado no objetivo especifico $(\mathrm{g})$ anteriormente apresentado. Dirige-se particularmente aos profissionais, incluindo os do CAFAP, e às instituições comunitárias que trabalham nas áreas da família, infância e juventude, e que se constituem como população-alvo estratégica ou intermédia, através da qual é possível atingir a população-alvo final dos CAFAP, ou seja, famílias com crianças e jovens maltratadas e em situação de risco.

O funcionamento deste eixo está particularmente voltado para a melhoria da capacidade técnica para promover o bem-estar das crianças e jovens e o fortalecimento das famílias e para a criação e partilha de recursos materiais de apoio às famílias. $\mathrm{O}$ trabalho em parceria é essencial para o funcionamento deste eixo, desenvolvendo-se ações que visam melhorar o conhecimento da realidade local bem como os recursos teóricos e práticos ao dispor dos profissionais. $\mathrm{O}$ apoio para o desenvolvimento da capacidade técnica passa também pela prestação de serviços de consultoria para o desenvolvimento e implementação de programas diversos de intervenção e fortalecimento familiar. Por outro lado, pretende-se envolver a comunidade na disponibilização de um conjunto de recursos de apoio às famílias, procurando estimular-se a partilha não só entre profissionais e instituições, mas também entre famílias. O eixo 3 poderá contemplar as seguintes sub-estruturas:

Centro de Estudos. Poderão ser desenvolvidos pequenos estudos de avaliação de necessidades junto da comunidade e auscultações à mesma, tendo em vista o desenvolvimento de ações de intervenção familiar e comunitária que facilitem o alcance dos objectivos do
CAFAP. Os CAFAP podem colaborar com as Comissões Alargadas (das CPCJ), com as Redes Sociais ou outros organismos na dinamização de observatórios locais que permitam monitorizar a situação das crianças e das famílias e as suas necessidades. Podem ser efetuados pequenos estudos de caracterização da situação das famílias locais (em termos de composição, condições sociais de vida, variáveis relacionadas com o funcionamento familiar, com a utilização dos recursos da comunidade, com os níveis de participação social, com a situação escolar das crianças, com a qualidade da ocupação dos tempos livres, etc.), de levantamento de situações de risco, de monitorização dos recursos existentes, dos mecanismos de articulação entre instituições e características das intervenções em curso. As parcerias com o mundo acadêmico devem ser promovidas como forma de otimizar a produção de conhecimento que pode decorrer do trabalho com as famílias servidas pelo CAFAP. O conhecimento produzido pode informar a elaboração de planos de intervenção local integrados. Os CAFAP podem, ainda, dinamizar sessões de auscultação à população sobre as necessidades sentidas e propostas de melhoria das condições de vida das famílias. Por outro lado, podem ser desenvolvidos pequenos estudos de avaliação do processo e resultados das intervenções do CAFAP e de outros serviços, envolvendo-se os destinatários neste processo.

Centro de Consultoria e Formação. O CAFAP pode operar como um pequeno centro de formação local, promovendo ações, dinamizadas pela equipa ou por formadores convidados, dirigidas aos diferentes profissionais ou instituições que desenvolvem a sua atividade no âmbito da intervenção familiar, nomeadamente com famílias com crianças e jovens em risco. As ações a dinamizar podem ser informadas por levantamentos de necessidades efetuados pelo Centro de Estudos. Desta forma, a equipa do CAFAP pode apoiar outros profissionais para que estes possam levar a cabo projetos e ações de intervenção familiar, prestando serviços de consultoria para o desenvolvimento, implementação e avaliação de projectos diversificados. Os CAFAP podem ainda contribuir para a formação de profissionais através do acolhimento de estágios curriculares e profissionais, aumentado, ao mesmo tempo, a capacidade de resposta do serviço.

Centros de Recursos. É possível constituir um pequeno centro de recursos técnicos que permita a disponibilização de recursos bibliográficos e/ou educativos (materiais para a intervenção; instrumentos de avaliação) que possa ser partilhado (co-organizado e reforçado) com outros profissionais. Pode ser igualmente constituído um centro de recursos para a comunidade, com materiais informativos, lúdicos e pedagógicos que podem ser requisitados pelas famílias, podendo igual- 
mente ser estimulada a partilha de recursos e serviços entre as famílias servidas pelo CAFAP e outras.

Outros Serviços. Neste âmbito podem ser desenvolvidas atividades que apoiem os profissionais no desenrolar do trabalho realizado nos eixos 1 e 2, por exemplo, através da dinamização de serviços de recolha e atribuição de gêneros alimentares, mobiliário e equipamentos domésticos, materiais escolares, roupa ou brinquedos. Dependendo dos recursos e instituições existentes na área geográfica abrangida pelo CAFAP é possível que, nalguns casos, se sinta a necessidade de oferecer alguns serviços adicionais que, de qualquer modo, devem ter um peso menor no conjunto das actividades do CAFAP. Estes serviços podem, ainda, incluir acompanhamento pedagógico, psicoterapia ou aconselhamento individual ou familiar focalizado num problema específico, serviços de mediação familiar, consulta para agressores, e assim por diante. Uma vez que outras respostas sociais, como os centros comunitários, serviços de atendimento/aconselhamento ou núcleos de atendimento a vítimas, podem prestar este tipo de serviços, os CAFAP devem disponibilizá-los apenas no caso de estas respostas não estarem disponíveis na comunidade local e perante situações de evidente necessidade, considerando-os situações de exceção.

\section{Eixo 4: Parcerias comunitárias e promoção da participação comunitária}

Finalmente, o eixo 4, procura dar resposta aos objetivos especificos (h) e (i) anteriormente apresentados, considerando como população-alvo específica a comunidade em geral.

O trabalho desenvolvido neste eixo é particularmente indicado quando nas preocupações da comunidade se insere a promoção do desenvolvimento positivo da criança, do jovem e o fortalecimento das famílias e da comunidade.

Neste eixo as intervenções são desenvolvidas em colaboração e de forma partilhada com a comunidade, seja com a população em geral, com famílias com crianças em risco ou com a comunidade técnica. Deste modo, a própria participação, das famílias abrangidas pelo CAFAP, em projetos que visem avaliar necessidades das comunidades relativamente à promoção do bem-estar das suas e de outras famílias, planificar, desenvolver e implementar ações de intervenção familiar e comunitária pode promover a ativação de recursos e competências familiares bem como facilitar o alargamento da rede de suporte social e, por conseguinte, contribuir para o fortalecimento da família. Espera-se, então, que a população-alvo final esteja também ela envolvida na planificação e na implementação das atividades. As intervenções deste eixo podem almejar sensibilizar a comunidade para as questões da promoção e proteção dos direitos das crianças, envolvê-la na criação ou rentabilização dos recursos existentes, na criação de redes de suporte formal e informal, para a participação cívica, entre outras questões. Neste eixo, distinguem-se duas grandes categorias de atividades que se podem cruzar em determinados momentos:

Intervenções com a Comunidade. A atuação do CAFAP deve ser conduzida de forma a dar poder às famílias e a envolvê-las mais ativamente na comunidade, promovendo a sua capacidade de adaptação, crescimento e fortalecimento. Neste âmbito podem ser desenvolvidas ações que visem aumentar a participação cívica das famílias abrangidas pelo serviço e o envolvimento comunitário de outras famílias e instituições. O CAFAP deve incentivar a partilha de recursos entre as famílias e diferentes elementos da comunidade e estimular o desenvolvimento de recursos que mais facilmente lhes permitam (re)negociar a sua posição social e encontrar apoio para o seu desenvolvimento. O serviço pode contribuir para a criação de fóruns comunitários e momentos de encontro em que possam construir-se soluções para melhorar as condições de vida de uma dada população, estimulando a construção de discursos sociais e relações comunitárias colaborativas nutritivas e apoiantes (Frankel, 2006).

Voluntariado. A equipa do CAFAP pode estimular e gerir a criação de grupos de voluntários, técnicos e não técnicos, que, devidamente formados e supervisionados, podem contribuir para a multiplicação e extensão das suas ações de apoio às famílias. Ao mesmo tempo, numa lógica de empowerment, as próprias famílias podem ser incentivadas a desenvolver atividades voluntárias em favor de outros e, ao mesmo tempo, desenvolver e reforçar competências promotoras da sua autonomia.

\section{Conclusão}

Os CAFAP podem funcionar como um recurso eficaz de apoio à família, particularmente famílias com crianças e jovens em situação de risco ou maltratadas e a todas as instituições e serviços (e.g., Tribunais e CPCJs) que desenvolvem esforços no sentido de aumentar a segurança, proteção e bem-estar das famílias e suas crianças e jovens. O modelo apresentado oferece uma estrutura de enquadramento das atividades dos CAFAP que poderá ser adaptada consoante as necessidades específicas de cada comunidade e, acima de tudo, um conjunto de linhas orientadoras e enfoques que, independentemente da organização adotada por cada instituição, se julga poder otimizar o trabalho dos mesmos. Espera-se que a proposta descrita possa criar uma base a partir da qual possam ser desenvolvidos e conjugados 
esforços de avaliação e investigação com enfoques diversificados, procurando desta forma responder aos desafios lançados por Biglan e Smolkowski (2002) no sentido da construção de comunidades, famílias, crianças e jovens mais fortes.

\section{Nota}

${ }^{1} \mathrm{O}$ presente trabalho foi desenvolvido no âmbito de uma bolsa de doutoramento financiada pela Fundação para a Ciência e Tecnologia (SFRH/BD/39912/2007), atribuída à primeira autora.

Agradece-se ao Gabinete de Atendimento à Família, em Viana do Castelo, e ao seu CAFAP, para o qual foram desenhados os primeiros esboços do modelo apresentado.

\section{Referências bibliográficas}

Asen, E. (2002). Multiple family therapy: An overview. Journal of Family Therapy, 24, 3-16.

Barlow, J., Johnston, I., Kendrick, D., Polnay, L., \& StewartBrown, S. (2006). Individual and group-based parenting programmes for the treatment of physical abuse and neglect. Cochrane Database of Systematic Reviews,3. Acesso em dezembro, 2007, em http://mrw.interscience.wiley.com/ cochrane/clsysrev/articles/CD005463/frame.html

Barth, R. P., Greeson, J. K. P., Guo, S., Green, R. L., Hurley, S., \& Sisson, J. (2007). Changes in family functioning and child behavior following intensive in-home therapy. Children and Youth Services Review, 29, 988-1009.

Berry, M., Charlson, R., \& Dawson, K. (2003). Promising practices in understanding and treating child neglect. Child and Family Social Work, 8, 13-24.

Biglan, A. \& Smolkowski, K. (2002). The role of the community psychologist in the $21^{\text {st }}$ century. Prevention and Treatment, 5(1), Article 2. Acesso em 16 de fevereiro, 2003, em http:// journals.apa.org/prevention/volume5/pre0050002a.html

Bronfenbrenner, U. (1979). The ecology of human development: Experiments by nature and design. Cambridge, MA: Harvard University Press.

Bunting, L. (2004). Parenting programmes: The best available evidence. Child Care in Practice, 10(4), 327-343.

Carr, A. (2000). Evidence-based practice in systemic consultation I. Child-focused problems. Journal of Family Therapy, 22, 29-60.

Chaffin, M. \& Friedrich, B. (2004). Evidence-based treatments in child abuse and neglect. Children and Youth Services Review, 26, 1097-1113.

Cirillo, S. \& Di Blasio, P. (1997). Niños maltratados. Diagnóstico y terapia familiar. Barcelona: Paidós.

Costa, L. F. \& Brandão, S. N. (2005). Abordagem clínica no contexto comunitário: uma perspectiva integradora. Psicologia \& Sociedade, 17(2), 33-41.

Direcção Geral da Segurança Social, da Família e da Criança. (2006). Respostas Sociais-Nomeclaturas/Conceitos [Versão electrónica]. Lisboa: Autor.

Doherty, W. J. \& Beaton, J. M. (2000). Family therapist, community and civic renewal. Family Process, 39(2), 149-161.

Donohue, B. \& Van Hasselt, V. B. (1999). Development and description of an empirically based ecobehavioral treatment program for child maltreatment. Behavioral Interventions, 14, 55-82.

Dufour, S. \& Chamberland, C. (2004). The effectiveness of selected interventions for previous maltreatment: Enhancing the well-being of children who live at home. Child and Family Social Work, 9, 39-56.

Eyberg, S. M. (2003). Parent-child interaction therapy. In T. H. Ollendick \& C. S. Schroeder (Eds.), Encyclopedia of clinical child and pediatric psychology (pp. 446-447). New York: Plenum.

Fraenkel, P. (2006). Engaging families as experts: Collaborative family program development. Family Process, 45(2), 237-257.

Hillesheim, B. \& Cruz, L. R. (2008). Risco, vulnerabilidade e infância: algumas aproximações. Psicologia \& Sociedade, 20(2), 192-199.

Hoagwood, K. E. (2005). Family-based services in children's mental health: A research review and synthesis. Journal of Child Psychology and Psychiatry, 46(7), 690-713.

Hughes, R. (1994). A framework for developing family life education programs. Family Relations, 43(1), 74-80.

Kumpfer, K. L. \& Alvarado, R. (2003). Family-strengthening approaches for the prevention of youth problem behaviors. American Psychologist, 58(6-7), 457-465.

Lundahl, B. W, Nimer, J., \& Parsons, B. (2006). Preventing child abuse: A meta-analysis of parent training programs. Research on Social Work Practice, 16(3), 251-162.

Lutzker, J. R. \& Bigelow, K. M. (2002). Reducing child maltreatment. A guidebook for parent services. New York: The Guilford Press.

Manalo, V. \& Meezan, W. (2000). Toward building a typology for the evaluation of services in family support programs. Child Welfare, 79(4), 405-429.

Matos, R. A. \& Sousa, L. M. (2004). How multiproblem families try to find support in social services. Journal of Social Work Practice, 18(1), 65-80.

McCroskey, J. \& Meezan, W. (1998). Family-centered services: Approaches and effectiveness. Protecting Children from Abuse and Neglect, 8(1), 54-71.

Melo, A. \& Alarcão, M. (2008). Integrated family assessment and intervention model: A collaborative approach with multidefied families with at-risk children. Manuscript submitted to publication.

Mrakek, P. J. \& Haggery, R. J. (Eds.). (1994). Reducing risk for mental disorders: Frontiers for preventive intervention research. Washington, DC: National Academy Press.

Murray-Swank, A. B. \& Dixon, L. (2004). Family psychoeducation as an evidence-based practice. CNS Spectrum, 9-12, 905-912.

Portugal. (1999). Lei n. 147/99, de 1 de setembro. Lei de protecção de crianças e jovens em perigo. Diário da República, I Série-A, N. ${ }^{\circ}$ 204, 6115-6132.

Righthand, S., Kerr, B., \& Drach, K. (2003). Child maltreatment risk assessments. An evaluation guide. New York: The Haworth Maltreatment and Trauma Press.

Rojano, R. (2000). Terapia familiar comunitária. Perspectivas Sistemicas, 59. Acesso em dezembro, 2007, em http://www. redsistemica.com.ar/rojano.htm

Rojano, R. (2004). The practice of community family therapy. Family Process, 43, 59-77.

Rutter, M. (2005). Multiple meanings of a developmental perspective on psychopathology. European Journal of Developmental Psychology, 2(3), 221-252. 
Scannapieco, M. \& Connel-Carrick, K. (2005). Understanding child maltreatment. An ecological and developmental perspective. New York: Oxford University Press.

Taylor, T. K. \& Biglan, A. (1998). Behavioral family interventions for improving child-rearing: A review of the literature for clinicians and police makers. Clinical Child and Family Psychology Review, 1(1), 41-60.

Warren-Adamson, C. (2006). Research review: Family centres a review of the literature. Child and Family Social Work, 11, 171-182.

Weissberg, R. P., Kumpfer, K. L., \& Seligman, M. P. E. (2003). Prevention that works for children and youth. American Psychologist, 58(6-7), 425-432.

Werner, E. E. \& Smith, R. (1992). Overcoming the odds: High risk children from birth to adulthood. New York: Cornell University Press.

Ana Teixeira de Melo é psicóloga, mestre em Psicologia Clínica e aluna de doutoramento da Faculdade de Psicologia e de Ciências da Educação da Universidade de Coimbra e, presentemente bolseira pela Fundação para a Ciência e Tecnologia. Endereço para correspondência: Rua Camilo Vaz, $n^{\circ} 45,3^{\circ}$ Dto. Centro. 4430-686 Vila Nova de Gaia. Portugal. E-mail: anamelopsi@gmail.com

Madalena Alarcão é psicóloga, terapeuta familiar, e doutorada em Psicologia Clínica pela Universidade de Coimbra. É Professora Associada na Faculdade de Psicologia e de Ciências da Educação da Universidade de Coimbra.

E-mail: malarcao@fpce.uc.pt

Centros de Apoio Familiar e Aconselhamento Parental: proposta de um modelo global de organização

Ana Teixeira de Melo e Madalena Alarcão

Recebido: 12/10/2008

$1^{\text {a Revisão: } 13 / 03 / 2009}$

Aceite final: 23/04/2009 